\title{
OprD Protein Profile of Pseudomonas aeruginosa Isolates Resistant to Imipenem from Patients in Khartoum State - Sudan
}

\author{
Somaia Alsir ${ }^{1 *}$ and Omeima Salih ${ }^{2}$ \\ ${ }^{1}$ School of Pharmacy - Ahfad University for Women, Omdurman, Sudan \\ ${ }^{2}$ School of Health Sciences - Ahfad University for Women, Omdurman, Sudan \\ *Corresponding author
}

\begin{abstract}
A B S T R A C T
Keywords

Pseudomonas

aeruginosa,

Clinical isolates,

OprD, Sudan

Article Info

Accepted:

04 February 2019

Available Online:

10 March 2019

In Sudan, Pseudomonas aeruginosa is the most antibiotics resistant bacteria isolated among other bacterial strains of clinical impact. Carbapenems, such as imipenem are often used as last resort antibiotics for the treatment of multidrug-resistant Pseudomonas aeruginosa infection. The study was performed to evaluate the OprD porin protein profile among clinical isolates of Pseudomonas aeruginosa from urine samples. Fifty six clinical isolates of Pseudomonas aeruginosa were collected from different hospitals in Khartoum State. Imipenem susceptibility test was determined by the disk diffusion method. Carbapenems production was confirmed by Disk Enhancement Test (DET) and Combined disk test (CDT) Imipenem-Cloxacillin. The protein profile of the isolates was determined by SDS-poly acrylamide gel electrophoresis. Seventy two percent $(72 \%)$ of the isolates were resistant to imepenim and about forty percent $(39.9 \%)$ of the imepenium resistant isolates were metalllobeta lacatamases producers. However, all resistant isolates showed OprD prion protein deficiency. This concludes the importance of OprD protein expression in increasing the sensitivity of Pseudomonas aeruginosa to antibiotics.
\end{abstract}

\section{Introduction}

Pseudomonas aeruginosa is a Gram-negative opportunistic bacteria leading to nosocomial infections worldwide. In Sudan, Pseudomonas aeruginosa is considered the third causative agent of urinary tract infections particularly at Khartoum state (Mohamed Badri and Mohamed 2017). Furthermore, it is the most resistant bacteria isolated among other bacterial strains of clinical impact (Saeed et al., 2017). A study conducted on clinical isolates of Pseudomonas aeruginosa from patients at Khartoum state detected Metallo-
beta-Lactamase (MBL) genes VIM and IMP (Satir et al., 2016).

Carbapenems, such as imipenem and meropenem are often used as last resort antibiotics for the treatment of multidrugresistant Pseudomonas aeruginosa infections (Al-Bayssari et al., 2015). The main reported mechanism of resistance to imipenem involves the loss of OprD porin from the outer membrane proteins (OMPs) through deletions, mutations or insertions in the oprD gene (Liu 2018; Shariati et al., 2018). OprD is an outer membrane porin protein facilitating the 
permeation of basic amino acids, small peptides, and carbapenem antibiotics (Hancock et al., 1990). In this study we compare the OprD porin protein profile among clinical isolates of Pseudomonas aeruginosa from urine samples.

\section{Materials and Methods}

\section{Clinical isolates of Pseudomonas aeruginosa}

A total number of 56 Pseudomonas aeruginosa isolates were collected from the diagnostic laboratories of five governmental hospitals in Khartoum State during the period July to October 2017. The isolates were identified using microbiological and biochemical methods, at the Microbiology laboratory of Ahfad University for Women, for confirmation. The reference strain Pseudomonas aeruginosa (ATCC 27853) was used as a control and standard for protein profiling.

\section{Antimicrobial Susceptibility Testing (AST)}

All confirmed Pseudomonas aeruginosa isolates were tested against impenenm (10 $\mathrm{mcg}$ ) and other antibiotics by the disk diffusion method. A Pseudomonas aeruginosa suspension of 0.5 McFarland standard was inoculated on Mueller Hinton agar (Oxoid Co. Ltd., U.K.) by swabbing. After drying, antibiotic disks (Bioanalyse, Ankara, Türkiye.) were placed on the plate and then incubated overnight at $37^{\circ} \mathrm{C}$. The inhibition zone diameters were interpreted according to the Clinical and Laboratory Standards Institute (CLSI 2014) recommendations.

\section{Disk Enhancement Test (DET)}

The test was performed as described by Yong et al., 2002 for the differentiation of metallo$\beta$-lactamase (MBLs) producing clinical isolates of Pseudomonas aeruginosa. This phenotypic method is based on the specific inhibition of MBLs, which are enzyme's zinc dependence, by EDTA as a chelating agent. A $0.5 \mathrm{M}$ EDTA solution was prepared by dissolving $186.1 \mathrm{~g}$ of disodium EDTA. $2 \mathrm{H}_{2} \mathrm{O}$ in $1,000 \mathrm{ml}$ of distilled water. The $\mathrm{pH}$ was adjusted to 8.0 by using $\mathrm{NaOH}$ and was sterilized by autoclaving. Pseudomonas aeruginosa clinical isolates and standard strain were inoculated on Mueller Hinton agar plates as recommended by CLSI (Wayne 2014). Two $10 \mu \mathrm{g}$ imipenem disks were placed on the plate, and $10 \mu \mathrm{L}$ of EDTA solution was added to one of them to obtain the desired concentration $(750 \mu \mathrm{g})$. The inhibition zones of the imipenem and imipenem-EDTA disks were compared after 18 hours of incubation at $37^{\circ} \mathrm{C}$. An increase of $\geq 7 \mathrm{~mm}$ in zone inhibition diameter around the imipenem and EDTA disk in comparison to the imipenem disk alone was interpreted as a positive result for MBL production.

\section{Combined disk test (CDT) Imipenem- Cloxacillin}

The test was done as described by Ahmed et al., 2017 to screen for OprD-deficient strains thus discriminating carbapenemase producing Pseudomonas aeruginosa strains from nonproducers. The CDT is based on the observation that imipenem resistance resulting from OprD deficiency requires constitutive and/or carbapenem-induced overproduction of $\mathrm{AmpC}$, therefore inhibition of $\mathrm{AmpC}$ by cloxacillin is expected to restore partial or complete sensitivity to imipenem in OprDdeficient strains but not in carbapenemase positive strains.

A 0.5 McFarland suspension from each isolate and reference strain were inoculated on Muller Hinton agar plate as recommended by CLSI (Wayne 2014). Two disks were placed on the Muller Hinton agar plate for each isolate as follows: a 10- $\mu \mathrm{g}$ Imipenem disk and a $10-\mu \mathrm{g}$ 
Imipenem disk supplemented with a cloxacillin load of $400 \mathrm{mg} / \mathrm{ml}$ with an end concentration of $4,000 \mu \mathrm{g}$ per disk. After $20 \mathrm{~h}$ of incubation at $37^{\circ} \mathrm{C}$, the difference between the zone diameters around imipenem disk alone and disk supplemented with cloxacillin at concentration of $4,000 \mu \mathrm{g}$ was measured in millimeters. A cutoff value of $5 \mathrm{~mm}$ is considered where OprD-deficient strains showed an increase in the zone size of $>5 \mathrm{~mm}$ for imipenem in the presence of cloxacillin compared with that of the drug alone while OprD strains were $<5 \mathrm{~mm}$.

\section{Outer membrane proteins analysis}

The method used is a combination of Ocampo-Sosa et al., 2012 and Meenakshisundaram et al., 2015. Cultures of Pseudomonas aeruginosa were grown overnight at $37^{\circ} \mathrm{C}$ in $5 \mathrm{ml}$ of Mueller-Hinton Broth medium (Difco/Becton Dickinson, Sparks, MD) and then diluted 100-fold into fresh medium. Bacterial cells were incubated for approximately $5 \mathrm{~h}$ with shaking at $37^{\circ} \mathrm{C}$ to yield late- logarithmic-phase cells. Outer membrane proteins (OMP) were extracted from logarithmic-phase cultures using a previously reported method (Meenakshisundaram et al., 2015).

The OMPs were profiled by sodium dodecyl sulfate-polyacrylamide gel electrophoresis by running on a standard $12 \%$ sodium dodecyl sulfate (SDS)-polyacrylamide gels and stained with Coomassie blue. OprD profiles from clinical isolates were compared with the reference strain Pseudomonas aeruginosa (ATCC 27853) protein profile.

\section{Results and Discussion}

All 56 isolates were confirmed by microbiological and biochemical tests to be Pseudomonas aeruginosa. Seventy two percent $(72 \%)$ of the isolates were resistant to imepenim while the rest of the isolates (28\%) were susceptible. Table 1 summarizes the phenotypic characteristics of the different isolates based on the results of the disk enhancement test and combined disk test.

About forty percent (39.9\%) of the imepenim resistant isolates are MBL producers, out of which $31 \%$ are OrpD-deficient. Eighteen percent (25\% of the whole number of isolates) of the imepenim resistant isolates are nonMBL producers but are OrpD-deficient. That result in $49 \%$ of the resistant isolates are OrpD-deficient.

All isolates were analyzed for OrpD protein expression out of which only eight isolates of Pseudomonas aeruginosa OMPs profile is presented in figure 1 . The isolates displayed selected to reflect there was an obvious discrepancy in the expression of the OrpD protein between the different Pseudomonas aeruginosa isolates. The isolates whose protein profiles are on lanes 1, 3, 4 and 7 were impeniem resistant and OrpD-deficient according to the Imipenem-Cloxacillin CDT phenotypic test.

All these four isolates did not express the OrpD prion protein except for the isolate on lane 4 which showed weakly expressed OrpD prion protein of molecular weight $45-\sim 49$ $\mathrm{kDa}$ (Schiavano et al., 2017). Isolate in lane 2 was positive by Imipenem-Cloxacillin CDT at conc. 4000 denoting OprD-deficient strain and was not MBL producer.

The isolates in lane 4 and 3 were also resistant to impeniem and both were MBL producers but were expressing OrpD prion protein with different levels in reference to the standard strain of Pseudomonas aeruginosa. The isolate in lane 1 was susceptible to impeniem and was neither MBL producer nor OrpD-defiient. The isolate in lane 1 resembles the standard strain of Pseudomonas aeruginosa in most of its protein profile. 
Table.1 Imipenem susceptibility and phenotypic results of Pseudomonas aeruginosa clinical isolates

\begin{tabular}{|c|c|c|c|}
\hline $\begin{array}{c}\text { Percent of } \\
\text { Isolates }\end{array}$ & $\begin{array}{c}\text { Imipenem Susceptibility } \\
\text { Test }\end{array}$ & $\begin{array}{c}\text { Imipenem-EDTA Double Disk } \\
\text { Synergy Test }\end{array}$ & $\begin{array}{c}\text { Combined Disk Test } \\
\text { Imipenem-Cloxacillin (4000 ug) }\end{array}$ \\
\hline $\mathbf{3 1 \%}$ & $\mathrm{R}$ & POS & POS \\
\hline $\mathbf{2 5 \%}$ & $\mathrm{R}$ & NEG & POS \\
\hline $\mathbf{8 . 9 \%}$ & $\mathrm{R}$ & POS & NEG \\
\hline $\mathbf{7 . 1 \%}$ & $\mathrm{R}$ & NEG & NEG \\
\hline $\mathbf{2 8 \%}$ & $\mathrm{S}$ & NEG & NEG \\
\hline ATCC 27853 & $\mathrm{S}$ & NEG & NEG \\
\hline
\end{tabular}

Fig.1 SDS-PAGE of cell proteins extracted from Pseudomonas aeruginosa isolates

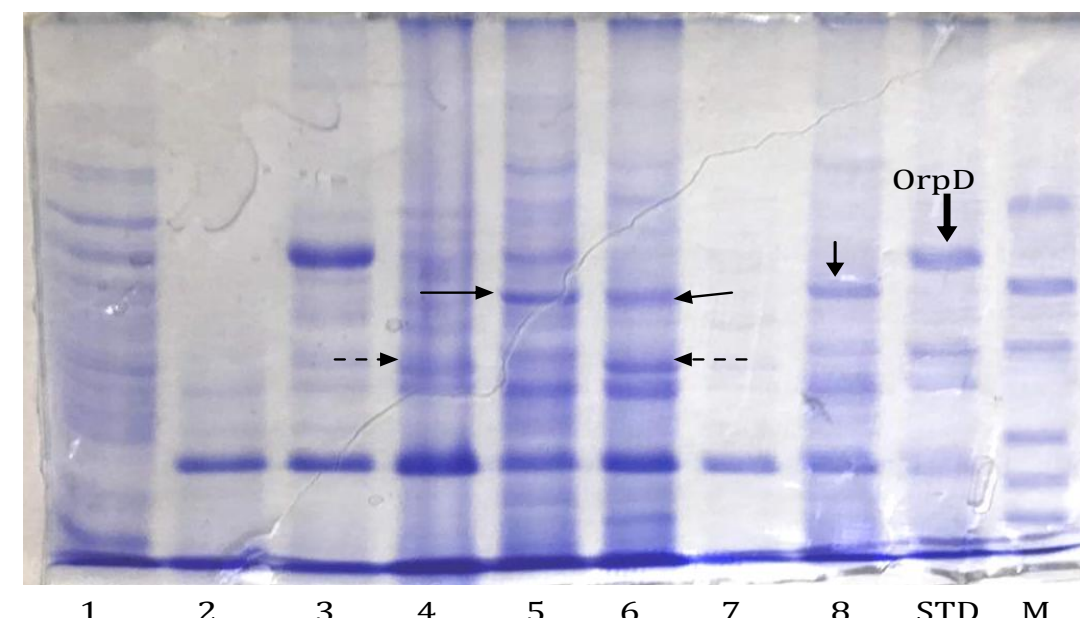

$51 \mathrm{KDa}$

$42 \mathrm{KDa}$

$29 \mathrm{KDa}$

$22 \mathrm{KDa}$

$14 \mathrm{KDa}$

$10.5 \mathrm{KDa}$

Lane 1, 3 : Imipenem susceptible (IMP(S)),DET(-),CDT(+)

Lane 2, 5 : Imipenem resistance (IMP(R)) DET(-) CDT(+)

Lane 4 : IMP(R), DET(-), CDT(-)

Lane 6, $8: \operatorname{IMP}(\mathrm{R}), \operatorname{DET}(+), \operatorname{CDT}(+)$

Lane 7 : IMP(R), DET(+), CDT(-)

STD : standard strain

M : Protein marker 
The occurrence of multi-drug resistant Pseudomonas aeruginosa isolates among the samples collected from different hospitals in Khartoum state reflects a serious treatment challenge. Seventy two percent $(72 \%)$ of the isolates were resistant to imepenim. This result disagrees with previous study from Sudan, which stated that all Pseudomonas aeruginosa strains $(n=67)$ from hospitals were found sensitive (82.1- 100\%) when tested against gentamicin, amikacin, ceftazidime, impenem and ciprofloxacin. However, the prevalence of MBL producing Gram- negative bacilli has increased in some hospitals, particularly among clinical isolates of Pseudomonas aeruginosa (Mukhtar and Saeed 2011). Carbapenems exhibit a broader spectrum of antibacterial activity towards Gram-positive and Gramnegative bacteria than other beta-lactams. However, twenty six percent (26\%) of the isolates were resistant to all antimicrobials, carbapenemases producers and OprD-deficient. Carbapenemases are versatile $\beta$-lactamases that have the ability to hydrolyse penicillin, cephalosporin, and monobactams. Resistance to carbapenems, is caused mainly by carbapenemase production or by porin loss or combined with the expression of betalactamases. All resistant isolates of Pseudomonas aeruginosa are OrpD prion protein deficient. The Pseudomonas aeruginosa porin OprD is a substrate-specific porin that facilitates the diffusion of basic amino acids, small peptides, and carbapenems into the cell. OprD mediated resistance occurs as a result of decreased transcriptional expression of oprD and/or function mutations that disrupt protein activity. The carbapenems, meropenem, ertapenem, and doripenem are substrates of the efflux pumps, whereas imipenem is not. Therefore mutations leading to the upregulation of the MexAB-OprM active efflux system may increase the resistance to meropenem, while imipenem is not affected by this route.

In this study $61 \%$ of Pseudomonas aeruginosa isolates are phenotypically tested as OrpD deficient. Although down regulation of the OprD porin alone is a source of intermediate susceptibility or resistance to imipenem, it decreases the susceptibility to a lesser extent to meropenem in Pseudomonas aeruginosa.

Acquired carbapenem resistance due to the production of MBLs has been increasingly reported in Pseudomonas spp. In this study 53\% of the isolates are MBLs producers. The prevalence of Pseudomonas spp that produce MBLs can be markedly different in distinct geographical areas, even among different hospitals in the same area. In Turkey, the prevalence of $P$. aeruginosa that produce MBLs were reported as between $10 \%$ and $56.8 \%$ in previous studies (Yilmaz et al., 2014).

Imipenem resistance can involve low permeability, the activity of an inducible $\beta$ lactamase, and multidrug efflux systems, but the most common mechanism underlying resistance involves the loss of OprD porins from the outer membrane, which can occur at the transcriptional or translational level or through the emergence of mutations in the oprD gene (Pirnay et al., 2002). In this study 66\% of the Pseudomonas aeruginosa isolates are Imipenem resistant. This was clearly correlated to the absence of OprD protein expression as reflected by the SDS-PAGE protein analysis.

\section{References}

Ahmed, O.M., Manal, A.A. and Samia, A.G., 2017. Evaluation of a New Phenotypic Method to Screen for OprD- Deficient M utant Strains of Pseudomonas aeruginosa., 6(2), pp.18941901.

Al-Bayssari, C., Valentini, C., Gomez, C., Reynaud-Gaubert, M., and Rolain, J. M. (2015). First detection of insertion sequence element ISPa1328 in the oprD porin gene of an imipenem-resistant Pseudomonas aeruginosa isolate from an idiopathic pulmonary fibrosis patient in Marseille, France. New microbes and new infections, 7, 26-7. doi:10.1016/j.nmni.2015.05.004

CLSI. 2014. Performance standards for antimicrobial susceptibility testing; 24th informational supplement. CLSI document M100-S24. Wayne, PA: Clinical and 
Laboratory Standards Institute.

Hancock, R.E., Siehnel, R. and Martin, N., 1990. Outer membrane proteins of Pseudomonas. Molecular Microbiology, 4, pp.1069-1075.

Liu, H., Kong, W., Yang, W., Chen, G., Liang, H., and Zhang, Y. (2018). Multilocus sequence typing and variations in the oprD gene of Pseudomonas aeruginosa isolated from a hospital in China. Infection and Drug Resistance, 11, 45-54. http://doi.org/10.2147/ IDR.S152162 accessed on $4^{\text {th }}$ of April 2018.

Meenakshisundaram, C., P. Rajendran, Usha Anand Rao, V. Mohan, and R. Vasudevan..2015. Original Research Article Whole Cell Protein Profiles of Pseudomonas aeruginosa Strains Isolated at a Tertiary Care Diabetic Specialty Hospital in Chennai, Tamilnadu, India., 4(9), pp.241-250.

Mohamed Badri, A. and Mohamed, S.G., 2017. Clinical Epidemiology and Antibiogram of UTI Patients Attended Different Hospital in Khartoum, Sudan. Clinical Microbiology: Open Access, 6(5).

Mukhtar, A.M. and Saeed, H.A., 2011. Profile of Antibiotic Sensitivity and Resistance of Some Pathogenic Bacteria Isolated from Clinical Specimens in Sudan. J.Sc. Tech, 12(1), pp.14 19.

Ocampo-Sosa, A. A., Cabot, G., Rodríguez, C., Roman, E., Tubau, F., Macia, M. D., Moya, B., Zamorano, L., Suárez, C., Peña, C., Domínguez, M. A., Moncalián, G., Oliver, A., Martínez-Martínez, L., 2012.Alterations of OprD in carbapenem-intermediate and susceptible strains of Pseudomonas aeruginosa isolated from patients with bacteremia in a Spanish multicenter study. Antimicrobial agents and chemotherapy, 56(4), 1703-13.

Pirnay, J.P., De Vos, D., Mossialos, D., Vanderkelen, A.,Cornelis, P., and Zizi, M. 2002. Analysis of the Pseudomonas aeruginosa oprD gene from clinical and environmental isolates. Environ Microbiol doi: 10.1046/j.1462-2920.2002.00281.x.
Saeed, A., Hamid, S. A., Bayoumi, M., Shanan, S., Alouffi, S., Alharbi, S. A., Alshammari, F.D., Abd, H. (2017). Elevated antibiotic resistance of Sudanese urinary tract infection bacteria. EXCLI journal, 16, 1073-1080. doi:10.17179/excli2017-424

Satir, S. B., Elkhalifa A. I, Ali M. A., El Hussein A., E.I.M. and E.K.A., 2016. Detection of Carbepenem Resistance Genes among Selected Gram Negative Bacteria Isolated from Patients in -Khartoum... Clinical Microbiology: Open Access Detection of Carbepenem resistance genes among selected Gram Negative bacteria isolated from patient., 5(November).

Schiavano, G. F., Carloni, E., Andreoni, F., Magi, S., Chironna, M., Brandi, G., and Amagliani, G. (2017). Prevalence and antibiotic resistance of Pseudomonas aeruginosa in water samples in central Italy and molecular characterization of oprD in imipenem resistant isolates. PloS one, 12(12), e0189172. doi:10.1371/journal.pone.0189172

Shariati, A., Azimi, T., Ardebili, A., Chirani, A. S., Bahramian, A., Pormohammad, A., Sadredinamin, M., Erfanimanesh, S., Bostanghadiri, N., Shams, S.,Hashemi, A. 2017. Insertional inactivation of oprD in carbapenem-resistant Pseudomonas aeruginosa strains isolated from burn patients in Tehran, Iran. New microbes and new infections, 21, 75-80. doi:10.1016/j.nmni. 2017.10.013

Yilmaz N O, Agus N, Bozcal E, Uzel A. 2014. Prevalence and molecular characterization of metallo-beta-lactamase producing strains of imipenem-resistant Pseudomonas aeruginosa in Turkey. Indian J Med Microbiol 32: 349350.

Yong D., Lee K., Yum J. H., Shin H. B., Rossolini G. M., Chong Y. 2002. Imipenem EDTA disc method for differentiation of metallo beta lactamase producing clinical isolates of Pseudomonas spp and Acinetobacter spp. J Clin Microbiol 40, pp. 3798-3801.

\section{How to cite this article:}

Somaia Alsir and Omeima Salih. 2019. OprD Protein Profile of Pseudomonas aeruginosa Isolates Resistant to Imipenem from Patients in Khartoum State - Sudan. Int.J.Curr.Microbiol.App.Sci. 8(03): 1-6. doi: https://doi.org/10.20546/ijcmas.2019.803.001 